\title{
Quantum phase transitions in the one-dimensional asymmetric Hubbard model: a bosonization study
}

\author{
Z. G. Wang ${ }^{1,2}$, Y. G. Chen ${ }^{1}$, and S. J. Gu² \\ 1 Department of Physics, Tongji University, Shanghai, 200092, China and \\ 2 Department of Physics and the Institute of Theoretical Physics, \\ the Chinese University of Hong Kong, Hong Kong, China
}

(Dated: October 26, 2018)

\begin{abstract}
The quantum phase transitions in the one-dimensional asymmetric Hubbard model are investigated with the bosonization approach. The critical conditions for the transition from density wave to phase separation, the correlation functions and their exponents are obtained analytically. Our results show that the difference between the hopping integrals for up- and down-spin electrons is crucial for the happening of the phase separation. When the difference is large enough, the phase separation will appear even if the on-site interaction is small.

PACS numbers: 71.10.Fd, 03.75.Mn, 05.70.Jk
\end{abstract}

\section{INTRODUCTION}

The Hubbard model $(\mathrm{HM})^{1}$ is one of the simplest nontrivial models of interacting spin- $1 / 2$ electrons on a lattice. Its Hamiltonian reads

$$
H_{\mathrm{Hub}}=-t \sum_{j=1}^{L} \sum_{\delta= \pm 1} c_{j, \sigma}^{\dagger} c_{j+\delta, \sigma}+U \sum_{j} n_{j \uparrow} n_{j \downarrow},
$$

where $c_{j, \sigma}^{\dagger}$ and $c_{j, \sigma}, \sigma=\uparrow, \downarrow$ are creation and annihilation operators for electrons with spin $\sigma$ at site $j$ respectively, $n_{\sigma}=c_{\sigma}^{\dagger} c_{\sigma}, t$ is the hoping integral, and $U$ denotes the strength of on-site interaction. In one dimension (1D) the HM can be solved exactly by the Bethe-ansatz method. ${ }^{2,3}$ The wave function and the energy of $N=N_{\uparrow}+N_{\downarrow}$ electrons on a chain with $L$ sites can be written in terms of $N$ pseudo-momentum variables and $N_{\downarrow}$ spin rapidities. Although the energy spectra have been known for many years, the calculation of the correlation functions proved to be a delicate problem $\underline{4,5,6.7}$ The numerical evaluations of the correlation functions ${ }^{5}$ and the analytic results 6 indicated clearly that the 1D HM is a Tomonaga-Luttinger liquid (TLL), 9.9 Assuming that the $1 \mathrm{D}$ HM is TLL, it then becomes possible to calculate the correlation functions from the knowledge of the energy spectra ${ }^{10}$. Using this procedure Schulz ${ }^{11}$ studied the correlation-function exponents for different $U$ and band filling $n$. It is also shown $^{12}$ that the large scale behavior of the spin and charge degrees of freedom can be described by two decoupled boson field theories with dynamics governed by the TLL Hamiltonian in the small and large $U$ regimes.

Another nontrivial model is the Falicov-Kimball model ${ }^{13}$ which consists of localized ions and itinerant spinless fermions. The Hamiltonian of the FalicovKimball model reads

$$
H_{\mathrm{FK}}=-t \sum_{j=1}^{L} \sum_{\delta= \pm 1} c_{j}^{\dagger} c_{j+\delta}+U \sum_{j} n_{j} w_{j}
$$

where $c_{j}^{\dagger}$ are creation operators for spinless fermions, and the configuration $\left\{w_{j}\right\}$ denote spatial distribution of ions. Clearly, the FKM can be viewed as a modification of the HM in the sense that the one kind of fermions, such as down-spin fermions, has infinite mass, and hence does not move. Nevertheless, the physics of the FKM is completely different. In the neutral case where each particle concentration equals $1 / 2$, it was ${ }^{14}$ proved that the system always orders in an alternating "chessboard" phase at a finite transition temperature in all dimensions greater than 1. This ordered phase can be interpreted as the transition from a high-temperature homogeneous (liquid/gas) phase to a low temperature ordered (solid) phase. Freericks ${ }^{15}$ showed that the model (on a hypercubic lattice) also displayed incommensurate order, segregation or phase separation. The 1D case of the FKM has also been extensively studied. Since there is no finitetemperature phase transition, the system can have phase transition in the ground state. The numerical solutions 16 produced a conjecture for the case $n_{e}+n_{i}<1$ with $n_{e}=N_{e} / N, n_{i}=N_{i} / N$ and the screened Coulomb interaction $U$ is large enough, the system will segregate into an empty lattice (with no ions and all the electrons), and a full lattice (with all the ions and no electrons). This conjecture was later proven to be true by Lemberger ${ }^{17}$. For any dimensional HFM, Freericks eta ${ }^{18}$ gave a theorem that the strong correlation can leads to PS.

The relation between the HM and the FKM is straightforward. In order to have a unified framework, the asymmetric Hubbard model (AHM) has been introduced naturally. 19,20,21,22 Its Hamiltonian reads

$$
H_{\mathrm{AHM}}=-\sum_{j=1}^{L} \sum_{\delta= \pm 1} \sum_{\sigma} t_{\sigma} c_{j, \sigma}^{\dagger} c_{j+\delta, \sigma}+U \sum_{j=1}^{L} n_{j, \uparrow} n_{j, \downarrow}
$$

where $t_{\sigma}$ is $\sigma$-dependent hoping integral. Clearly, if $t_{\uparrow}=t_{\downarrow}$, the AHM becomes the HM, and if $t_{\downarrow}=0$, it becomes the FKM. The Hamiltonian (3) has U(1) $\otimes \mathrm{U}(1)$ symmetry for general $t_{\sigma}$, and the electron number $N_{\downarrow}, N_{\uparrow}$ are conserved respectively. In the condensed matter physics, the AHM is believed to describe many physical phenomena, such as superconductor, valence fluctuating, and heavy fermions. ${ }^{23,24}$ In the recent development of the 
optical lattice, it has been pointed out that the AHM can be used to describe a mixture of two species of fermionic atoms in optical lattices..$^{25,26}$

According to the fact that the ground states of the Hamiltonian (3) in its two limiting cases: the HM $\left(t_{\uparrow}=\right.$ $\left.t_{\downarrow}\right)$ and the FKM $\left(t_{\downarrow}=0\right)$ belong to two different universality classes, a quantum phase transition was suggested to happen in the phase diagram defined in the $U-t_{\downarrow}$ plane $\stackrel{20,22,25}{2}$ Nevertheless, the quantitative phase diagram for the case away from half-filling had never been obtained until a recent work by $\mathrm{Gu}$, et $a^{26}$. In their work, the quantum entanglement ${ }^{27}$ between a local part and the rest of the system, and the structure factor of charge-density-wave (CDW) for down-spin electrons are used to identify the critical point. Here, we are going to study the ground-state phase diagram of the AHM away from the half filling with the strategy of bosonization method. $^{28}$ Differ from the numerical approach ${ }^{26}$ which captures the physics from the finite-size analysis for small systems, our work aim to give definitely analytical results from the point of view of field theory. The paper is organized as follows. In section II, we derive the bosonized form of the 1D AHM, and clarify the role of the some terms in the Hamiltonian. In section III we first diagonalize the effective Hamiltonian in which some irrelevant terms are ignored, then obtain the instability conditions for the phase separation and compare them with the numerical results of a finite sample. We also obtain the analytical expressions for the correlation functions of charge-density-wave, spin-density-wave (SDW), singlet-superconductivity (SS), triplet-superconductivity (TS) fluctuations, as well as the corresponding exponents. Finally, a brief summary and acknowledgement are given in section IV.

\section{THE BOSONIZED FORM OF ONE-DIMENSIONAL ASYMMETRIC HUBBARD MODEL}

The convenient way to analyze the $1 \mathrm{D}$ AHM is to bosonize the Fermi operators and convert them to a quantum theory of two Bose fields $\frac{28,29}{2}$. In the framework of the standard bosonization method the HM are expressed in terms of canonical Bose fields and their dual counterparts as,

$$
\begin{aligned}
H_{B}= & \frac{v_{c}}{2} \int d x\left[\frac{1}{K_{c}}\left(\partial_{x} \phi_{c}\right)^{2}+K_{c} \pi_{c}^{2}\right] \\
& +\frac{v_{s}}{2} \int d x\left[\frac{1}{K_{s}}\left(\partial_{x} \phi_{s}\right)^{2}+K_{s} \pi_{s}^{2}\right] \\
& +\delta v \int d x\left[\pi_{c} \pi_{s}+\partial_{x} \phi_{c} \partial_{x} \phi_{s}\right] \\
& +\frac{U}{2 \pi^{2} a} \int d x \cos \left(\sqrt{8 \pi} \phi_{s}\right) \\
& +\frac{U}{2 \pi^{2} a} \int d x \cos \left(\sqrt{8 \pi} \phi_{c}+2\left(k_{F \uparrow}+k_{F \uparrow}\right) x\right)
\end{aligned}
$$

with

$$
\begin{aligned}
v_{c} & =a \sqrt{t_{\uparrow} \sin \left(k_{F \uparrow} a\right)+t_{\downarrow} \sin \left(k_{F \downarrow} a\right)\left(t_{\uparrow} \sin \left(k_{F \uparrow} a\right)+t_{\downarrow} \sin \left(k_{F \downarrow} a\right)+\frac{U}{2 \pi}\right)}, \\
\frac{1}{K_{c}} & =\sqrt{1+\frac{U}{2 \pi\left[t_{\uparrow} \sin \left(k_{F \uparrow} a\right)+t_{\downarrow} \sin \left(k_{F \downarrow} a\right)\right]}}, \\
v_{s} & =a \sqrt{t_{\uparrow} \sin \left(k_{F \uparrow} a\right)+t_{\downarrow} \sin \left(k_{F \downarrow} a\right)\left(t_{\uparrow} \sin \left(k_{F \uparrow} a\right)+t_{\downarrow} \sin \left(k_{F \downarrow} a\right)-\frac{U}{2 \pi}\right)}, \\
\frac{1}{K_{s}} & =\sqrt{1-\frac{U}{2 \pi\left[t_{\uparrow} \sin \left(k_{F \uparrow} a\right)+t_{\downarrow} \sin \left(k_{F \downarrow} a\right)\right]}}, \\
\delta v & =a\left[t_{\uparrow} \sin \left(k_{F \uparrow} a\right)-t_{\downarrow} \sin \left(k_{F \downarrow} a\right)\right] .
\end{aligned}
$$

Here, the Bose field $\phi_{c}, \phi_{s}$ present the charge and spin degrees of freedom, respectively. $k_{F \uparrow}$ and $k_{F \downarrow}$ are the Fermi momentum for up- and down-spin electrons, $k_{F \uparrow}=$ $\pi n_{\uparrow} / a, k_{F \downarrow}=\pi n_{\downarrow} / a$, with $n_{\uparrow}=N_{\uparrow} / L$ and $n_{\downarrow}=N_{\downarrow} / L$ are the filling densities for up- and down-spin electrons, respectively; and $a$ lattice constant. $v_{c, s}$ are the propagation velocities of the charge and spin collective modes of the decoupled model $(\delta v=0)$, and $K_{c, s}$ are the stiffness constants.

It can be seen that this model is the standard HM at unpolarized case with both Bose fields decoupling. And if model is at half-filling band, $k_{F \uparrow}+k_{F \downarrow}=\pi / a$, the Umklapp term (the last term in $H_{B}$ ) is important and the HM is in the SDW phase with $U>0$ or in the CDW phase with $U<0$. As the model shifts away from the half-filling band, the Umklapp interaction can be can- 
celled. The difference of hopping integral and the filling densities of up- and down-spin electrons in the system appear as an effect that breaks the spin-charge separation as reveals the presence of the third term in the last equation.

Here, we discuss the quantum transition in the case of away from the half filling which means that the Umklapp interaction can be ignored. In general $t_{\uparrow}, t_{\downarrow}>0$. In the parameter region of $U>0$, since $K_{s}>1$, so the term of $\cos \left(\sqrt{8 \pi} \phi_{s}\right)$ is irrelevant whenever the system is at unpolarized case, in the one-loop approximation we can cancel it directly and get

$$
\begin{aligned}
H_{\text {Beff }}= & \frac{v_{c}}{2} \int d x\left[\frac{1}{K_{c}}\left(\partial_{x} \phi_{c}\right)^{2}+K_{c} \pi_{c}^{2}\right] \\
& +\frac{v_{s}}{2} \int d x\left[\frac{1}{K_{s}}\left(\partial_{x} \phi_{s}\right)^{2}+K_{s} \pi_{s}^{2}\right] \\
& +\delta v \int d x\left[\pi_{c} \pi_{s}+\partial_{x} \phi_{c} \partial_{x} \phi_{s}\right] .
\end{aligned}
$$

\section{RESULTS AND DISCUSSIONS}

The Hamiltonian (10) can be diagonalized in terms of two new phase fields which contains a mixture of spin and charge degrees of freedom. The propagation velocities of these collective modes are

$$
v_{ \pm}^{2}=\frac{v_{c}^{2}+v_{s}^{2}}{2}+\delta v^{2} \pm \sqrt{\left(\frac{v_{c}^{2}-v_{s}^{2}}{2}\right)^{2}+\delta v^{2}\left[v_{c}^{2}+v_{s}^{2}+v_{c} v_{s}\left(K_{c} K_{s}+\frac{1}{K_{c} K_{s}}\right)\right]} .
$$

As $\delta v \rightarrow 0, v_{+} \rightarrow \max \left(v_{c}, v_{s}\right)$ and $v_{-} \rightarrow \min \left(v_{c}, v_{s}\right)$. As $\delta v$ increases, $v_{-}$decreases until vanishes at the points

$$
\begin{aligned}
\delta v_{1}^{2} & =v_{c} v_{s} \frac{1}{K_{c} K_{s}} \\
\delta v_{2}^{2} & =v_{c} v_{s} K_{c} K_{s}
\end{aligned}
$$

At these points, the freezing of the lower bosonic mode is accompanied by a divergence in the charge and spin response functions. The static charge compressibility $\kappa$ diverges at $\delta v=\delta v_{1}$, or $\delta v=\delta v_{2}$. it behaves as

$$
\kappa=\kappa_{0}\left[1-\frac{\delta v}{\delta v_{1(2)}}\right]^{-1}, \quad \kappa_{0}=\frac{2 K_{c}}{\pi v_{c}}
$$

Beyond these points the susceptibilities becomes negatives. This behavior of the static response functions together with the vanishing of the collective modes velocity indicates that the system becomes unstable ${ }^{30}$ and undergoes a first order phase transition ${ }^{31}$. This instability is known as phase separation and has been shown to occur in the extended $\mathrm{HM}^{32,33}$ and in the $t-J$ model ${ }^{34,35}$.

In our case, we obtain

$$
\begin{aligned}
& \delta v_{1}=\sqrt{v_{c} v_{s} K_{c} K_{s}}=a t_{\uparrow} \sin \left(k_{F \uparrow} a\right)+t_{\downarrow} \sin \left(k_{F \downarrow} a\right), \\
& \delta v_{2}=\sqrt{\frac{v_{c} v_{s}}{K_{c} K_{s}}}=a t_{\uparrow} \sin \left(k_{F \uparrow} a\right)+t_{\downarrow} \sin \left(k_{F \downarrow} a\right) \sqrt{1-\left(\frac{U}{2 \pi\left(t_{\uparrow} \sin \left(k_{F \uparrow} a\right)+t_{\downarrow} \sin \left(k_{F \downarrow} a\right)\right)}\right)^{2}} .
\end{aligned}
$$

It is obvious that $\delta v_{1} \geq \delta v_{2}$, so the system is in PS phase state as

$$
\delta v \geq \delta v_{2},
$$

i.e.,

$$
\frac{t_{\uparrow} \sin \left(k_{F \uparrow} a\right)-t_{\downarrow} \sin \left(k_{F \downarrow} a\right)}{2} \geq \frac{t_{\uparrow} \sin \left(k_{F \uparrow} a\right)+t_{\downarrow} \sin \left(k_{F \downarrow} a\right)}{2} \sqrt{1-\left(\frac{U}{2 \pi\left(t_{\uparrow} \sin \left(k_{F \uparrow} a\right)+t_{\downarrow} \sin \left(k_{F \downarrow} a\right)\right)}\right)^{2}}
$$

Then we obtain the PS phase state condition

$$
\left(t_{\downarrow} \sin \left(k_{F \downarrow} a\right)\right)\left(t_{\uparrow} \sin \left(k_{F \uparrow} a\right)\right) \leq\left(\frac{U}{4 \pi}\right)^{2} .
$$

Let us now focus our attention on the correlation functions. Our interest in this work is observing the algebraic 
decay of the instantaneous correlation functions at zero temperature and studying how the exponents get modified from the standard HM. The operators for CDW, SDW, SS, and TS fluctuations in their bosonized form $\operatorname{are}^{10}$

$$
\begin{aligned}
& O_{\mathrm{CDW}}^{+}(x)=\Psi_{1 \uparrow}^{+} \Psi_{2 \uparrow} \\
& =\frac{1}{2 \pi \epsilon} \exp \left[\sqrt{2 \pi} i\left(\phi_{\rho}+\phi_{\sigma}\right)+2 i k_{F} x\right] \\
& O_{\mathrm{SDW}}^{+}(x)=\Psi_{1 \uparrow}^{+} \Psi_{2 \downarrow} \\
& =\frac{1}{2 \pi \epsilon} \exp \left[\sqrt{2 \pi} i\left(\phi_{\rho}(x)-\int_{-\infty}^{x} d y \Pi_{\sigma}(y)\right)-2 i k_{F} x\right] \\
& O_{\mathrm{SS}}^{+}(x)=\Psi_{1 \uparrow}^{+} \Psi_{2 \downarrow}^{+} \\
& =\frac{1}{2 \pi \epsilon} \exp \left[\sqrt{2 \pi} i\left(-\int_{-\infty}^{x} d y \Pi_{\rho}(y)+\phi_{\sigma}(x)\right)\right] \\
& O_{\mathrm{TS}}^{+}(x)=\Psi_{1 \uparrow}^{+} \Psi_{2 \uparrow}^{+} \\
& =\frac{1}{2 \pi \epsilon} \exp \left[\sqrt{2 \pi} i\left(-\int_{-\infty}^{x} d y \Pi_{\rho}(y)-\int_{-\infty}^{x} d y \Pi_{\sigma}(y)\right)\right]
\end{aligned}
$$

which represent fluctuations of CDW, SDW, SS and TS phases, respectively. The correlation functions are defined as

$$
R_{i}(x)=\left\langle: O_{i}(x) O_{i}^{+}(0):\right\rangle
$$

After some cacluation, we find that the correlation functions behavior as

$$
R_{i}(x) \sim|x|^{-2+\alpha_{i}}
$$

The exponents $\alpha_{i}$ 's determine the divergence of the corresponding phase. The expressions obtained for the $\alpha_{i}$ are

$$
\begin{aligned}
& \alpha_{\mathrm{CDW}}=2-K_{c} \nu^{c}-K_{s} \nu^{s}, \\
& \alpha_{\mathrm{SDW}}=2\left(1+|\gamma|^{s}\right)-K_{c} \nu^{c}-\mu^{s} / K_{s}, \\
& \alpha_{\mathrm{SS}}=2\left(1+|\gamma|^{c}\right)-\mu^{c} / K_{c}-K_{s} \nu^{s}, \\
& \alpha_{\mathrm{TS}}=2-\mu^{c} / K_{c}-\mu^{s} / K_{s} .
\end{aligned}
$$

with

$$
\begin{aligned}
& \mu_{c}=\frac{v_{c}}{v_{+}+v_{-}}\left[1+\frac{v_{s}^{2}}{v_{+} v_{-}}\left(1-\frac{\delta v^{2}}{\delta v_{2}^{2}}\right)\right], \\
& \mu_{s}=\frac{v_{s}}{v_{+}+v_{-}}\left[1+\frac{v_{c}^{2}}{v_{+} v_{-}}\left(1-\frac{\delta v^{2}}{\delta v_{1}^{2}}\right)\right], \\
& \nu_{c}=\frac{v_{c}}{v_{+}+v_{-}}\left[1+\frac{v_{s}^{2}}{v_{+} v_{-}}\left(1-\frac{\delta v^{2}}{\delta v_{1}^{2}}\right)\right], \\
& \nu_{s}=\frac{v_{s}}{v_{+}+v_{-}}\left[1+-\frac{v_{c}^{2}}{v_{+} v_{-}}\left(1-\frac{\delta v^{2}}{\delta v_{2}^{2}}\right)\right], \\
& \gamma_{c}=\frac{\delta v}{v_{+}+v_{-}}\left[1+\frac{\delta v_{2}^{2}-\delta v^{2}}{v_{+} v_{-}}\right], \\
& \gamma_{s}=\frac{\delta v}{v_{+}+v_{-}}\left[1+\frac{\delta v_{1}^{2}-\delta v^{2}}{v_{+} v_{-}}\right] .
\end{aligned}
$$

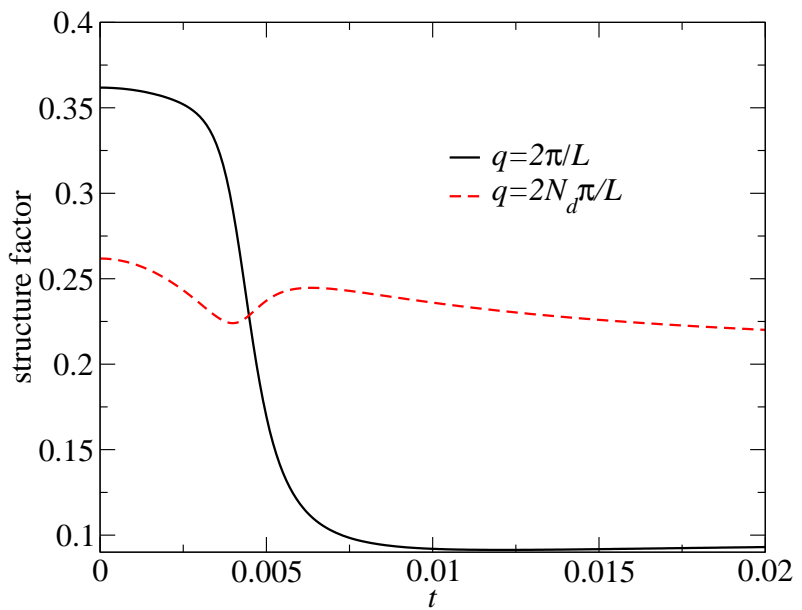

FIG. 1: (color online) The structure factor of the CDW for two different modes $q=\pi / L$ and $q=N_{\downarrow} \pi / L$ at given $U / t_{\uparrow}=$

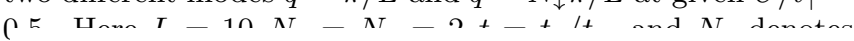

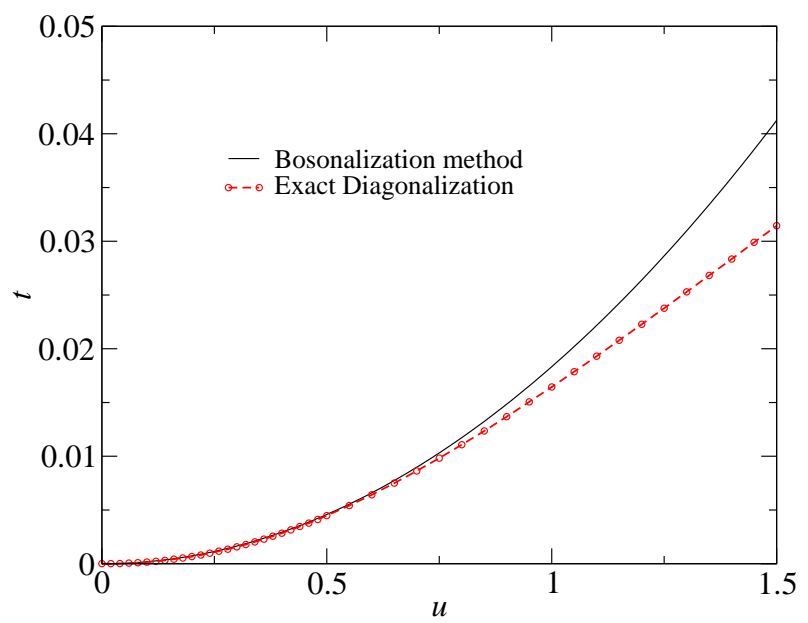

FIG. 2: (color online) The boundary line between the density wave and phase separation predicted by the bosonization method (solid line) and exact diagonalization (dotted line for $N=10, N_{\uparrow}=N_{\downarrow}=2$ ) at give concentration $n=2 / 5$. Here $t=t_{\downarrow} / t_{\uparrow}, u=U / t_{\uparrow}$.

The ground state is controlled and named by the most divergent correlation function, i.e., with the largest $\alpha_{i}$.

To have a deep understanding on the PS, it is very useful to study the structure factor of the CDW. Since the dominating configuration of electrons with spin-down is quite different in two phase, so we introduce the following structure of down-spin electrons,

$$
S_{\mathrm{CDW}}(q)=\frac{1}{L} \sum_{j l} e^{i q(j-l)}\left(\left\langle n_{j, \downarrow} n_{l, \downarrow}\right\rangle-\left\langle n_{\downarrow}\right\rangle^{2}\right),
$$

where $q=2 n \pi / L, n=0,1, \cdots, L$. We show the structure fact as a function of $t=t_{\downarrow} / t_{\uparrow}$ at a given $U / t_{\uparrow}=0.5$ for two different modes $q=\pi / L$ and $q=N_{\downarrow} \pi / L$ in Fig. [1] From the figure we find that in the small $t$ 


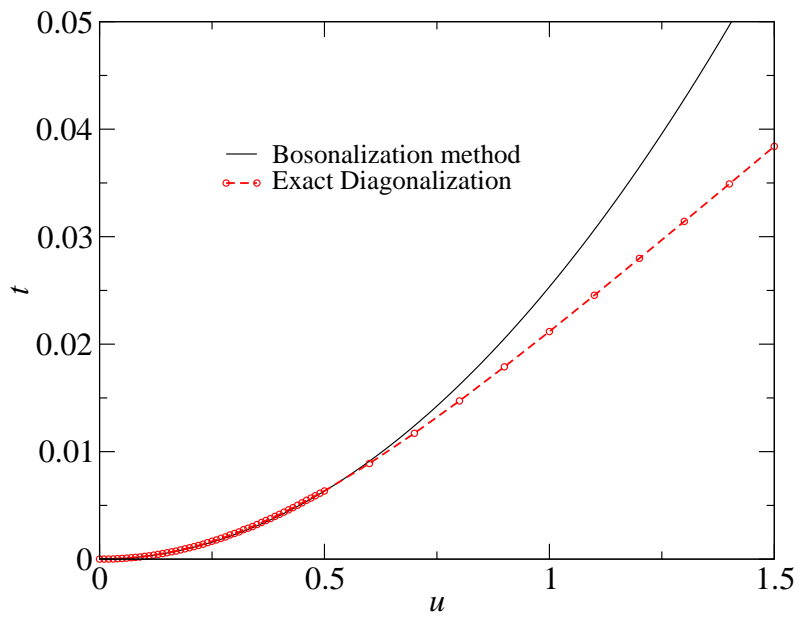

FIG. 3: (color online) The boundary line between the density wave and phase separation predicted by the bosonization method (solid line) and exact diagonalization (dotted line, $N=12, N_{\uparrow}=N_{\downarrow}=2$ ) at give concentration $n=1 / 3$. Here $t=t_{\downarrow} / t_{\uparrow}, u=U / t_{\uparrow}$.

limit, $S(\pi / L)$ dominate, this fact manifest the phase separation; while in a relatively larger $t$ region, $S\left(N_{\downarrow} \pi / L\right)$ dominates, this suggests the density-wave state. So we can use the intersection point of the structure factors of two different modes to determine the transition point. In Fig. 2 and 3 , we shown the phase diagram for different concentration $n=2 / 5,1 / 3$ in the small $U$ region. The results are very impressive. In both figures, we can see that if $U<0.5$, the numerical results from the exact diagonalization method agree with Eq. (19) excellently. That is the phase boundary in the small $U$ regime is proportional to $U^{2}$. However, when $U$ becomes large, say $U>1$, the bosonization results deviate from the numerical results apparently. We interpret it due to the fact the bosonization method becomes invalid in the large $U$ region. On the other hand, the excellent agreement between the results obtained from two approaches suggests that finite-size correction to the numerical data for a finite sample is very small.
Therefore, the bosonization results are wonderful in the small $U$ region and low concentration conditions. Since the 1D AHM is equivalent to the FKM if $t_{\downarrow}=0$ (or $t_{\uparrow}=0$ ), which has been proven that there exists PS phase at infinite- $U$ limit when the system shifts away form halffilling. From Eq. (19), we find that the PS phase always appears in the 1D FKM whenever the onsite interaction is small or large as the system shifts away form half-filling. On the other hand, the numerical studies ${ }^{26}$ suggest there might exist a critical $U$ if the density of electrons is close to half-filling. This inconsistence may due to the effect of Umklapp process around half-filling.

\section{SUMMARY}

In summary, we have studied the quantum phase transitions in the 1D AHM with the bosonization approach. In the framework of standard bosonization method, we first obtained an effective Hamiltonian of 1D AHM. Then we diagonalized the Hamiltonian and obtained the propagation velocities of the collective modes for both spin and charge degree of freedom. Based on the instability condition, we got the final critical conditions of the phase transition from DW to PS. We also obtained the analytical expressions for the correlation functions of CDW, SDW, SS, TS fluctuations, as well as the corresponding exponents.

Our results show that the difference between the hopping integrals for up- and down-spin electrons is crucial for the happening of the PS. When the difference is large enough, the phase separation will appear even if the on-site interaction is small. In the small- $U$ and low concentration region, critical conditions which scales like $t_{\downarrow} \propto U^{2}$ agree with the numerical results excellently.

We thank HQ Lin for suggesting this problem to us. SJ Gu is grateful for the hospitality of the Physics Department at Shanghai Jiaotong University. This work is supported by RGC Grant CUHK 401504, NSFC Grant No. 10304014, and and Foundations from Sci. \& Tech. Committee of Shanghai Municipality (Grant Nos. 03QA14054).
* Email:sjgu@phy.cuhk.edu.hk URL: http://www.phystar.net/

1 For example, The Hubbard Model - a reprint volume, edited by A. Montorsi (World Scientific, Singapore, 1992).

2 E. Lieb, F. Y. Wu, Phys. Rev. Lett. 20, 1445 (1968).

3 F. H. L. Essler, H. Frahm, F. Göhmann, A Klümper, and V. E. Korepin, The one-dimensional Hubbard model, (Cambridge 2005).

4 J. Carmelo and D. Baeriswyl, Phys. Rev. B 37, 7541 (1988).

5 M. Ogata, H. Shiba, Phys. Rev. B 41, 2326 (1990).

6 A. Parola, S. Sorella, Phys. Rev. Lett. 64, 1831 (1990); S. Sorella, A. Parola, M. Parrinello, and E. Tosatti, Europhys. Lett. 12, 721 (1990).
7 H. Frahm, V. E. Korepin, Phys. Rev. B 42, 10553 (1990); Phys. Rev. B 43, 5653 (1991).

8 S. Tomonaga, Prog. Theor. Phys. 5, 544 (1950); J. M. Luttinger, J. Math. Phys. 4, 1154 (1963).

9 F. D. M. Haldane, Phys. Rev. Lett. 45, 1358 (1980); 47, 1840 (1981); J. Phys. C 14, 2585 (1981).

10 J. Solyom and J. Timonen, Phys. Rev. B 40,7150 (1989); S. Coleman, Phys. Rev. D 11, 2088 (1975).

11 H. J. Schulz, Phys. Rev. Lett. 64, 2831 (1990).

12 K. Penc, J. Sólyom Phys. Rev. B 47, 6273 (1993).

13 L. M. Falicov and J. C. Kimball, Phys. Rev. Lett. 22, 997 (1969); R. Ramirez, L. M. Falicov, and J. C. Kimball, Phys. Rev. B 2, 3383 (1970); J. K. Freericks, Ch. Gruber and N. Macris, Phys. Rev. B 601617 (1999); J. K. Freericks and 
V. Zlatic, Rev. Mod. Phys. 75, 1333 (2003).

14 T. Kennedy and E. H. Lieb, Physica A 138, 320 (1986); U. Brandt and R. Schmidt, Z. Phys. B 63, 45 (1986).

15 J. K. Freericks, Phys. Rev. B 47, 9263 (1993); J. K. Freericks, Ch. Gruber and N. Macris, Phys. Rev B 60, 1617 (1999).

16 J. K. Freericks and L. M. Falicov, Phys. Rev. B 41, 2163 (1990).

17 P. Lemberger, J. Phys. A 25, 715 (1992).

18 J. K. Freericks, E. H. Lieb, and D. Ueltschi, Phys. Rev. Lett. 88, 106401 (2002).

19 U. Brandt, J. Low Temp. Phys. 84, 477 (1991).

${ }^{20}$ G. Fáth, Z. Domański, and R. Lemański, Phys. Rev. B 52, 13910 (1995); Z. Domański, and R. Lemański, G. Fáth, J. Phys. Condens. Matter 8, L261 (1996).

21 C. A. Macedo and A. M. C. de Souza, Phys. Rev. B 65, 153109 (2002).

22 D. Ueltschi, J. Stat. Phys. 116, 681 (2004).

23 V. J. Emery, Phys. Rev. Lett. 58, 2794 (1987).

24 C. M. Varma, Comm. Solid State Phys. 11, 221 (1985).

25 M. A. Cazalilla, A. F. Ho, and T. Giamarchi, Phys. Rev. Lett. 95, 226402 (2005).

26 S. J. Gu, R. Fan, and H. Q. Lin, arXiv: cond-mat/0601496
27 S. J. Gu, S. S. Deng, Y. Q. Li, and H. Q. Lin, Phys. Rev. Lett. 93, 086402 (2004); S. S. Deng, S. J. Gu, and H. Q. Lin, Phys. Rev. B 74, 045103 (2006).

${ }^{28}$ For a review of bosonization, see, e.g., V. J. Emery, in Highly Conducting One-Dimensional Solids, edited by J. T. Devreese, R. P. Evrard, and V. E. van Doren (Plenum, New York, 1979).

29 D. Shelton, A. A. Nersesyan, and A. M. Tsvelik, Phys. Rev. B 53, 8521 (1996).

30 J. Voit, Rep. Prog. Phys. 58, 977 (1995); J. Drut and D. Cabra, J. Phys.: Condens. Matter 15, 1445 (2003).

31 J. Voit, Phys. Rev. B 45, 4027 (1992).

32 H. Q. Lin, E. Gagliano, D. K. Campbell, E. H. Fradkin, and J. E. Gubernatis, in The Hubbard Model: Its Physics and Mathematical Physics, edited by D. Baeriswyl, pp. 315 (Plenum Press, 1995); see also H. Q. Lin, D. K. Campbell, and R. T. Clay, Chin. J. Phys. 38 , 1 (2000).

${ }^{33}$ K. Penc and F. Mila, Phys. Rev. B 49, 9670 (1994).

34 V. J. Emery, S. A. Kivelson, and H. Q. Lin, Phys. Rev. Lett. 64, 475 (1990).

35 M. Ogata, M. U. Luchini, S. Sorella, and F. F. Assaad, Phys. Rev. Lett. 66, 2388 (1991). 